10-7-2019

\title{
The Effects of Music Therapy on Individuals Suffering from Psychiatric Disorders: A Literature Review and Look into Future Prospects
}

\section{Zachary J. Krauss}

Cedarville University, zacharykrauss@cedarville.edu

Follow this and additional works at: https://digitalcommons.cedarville.edu/musicalofferings

Part of the Counseling Commons, Fine Arts Commons, Music Therapy Commons, and the Psychology Commons

DigitalCommons@Cedarville provides a publication platform for fully open access journals, which means that all articles are available on the Internet to all users immediately upon publication. However, the opinions and sentiments expressed by the authors of articles published in our journals do not necessarily indicate the endorsement or reflect the views of DigitalCommons@Cedarville, the Centennial Library, or Cedarville University and its employees. The authors are solely responsible for the content of their work. Please address questions to dc@cedarville.edu.

\section{Recommended Citation}

Krauss, Zachary J. (2019) "The Effects of Music Therapy on Individuals Suffering from Psychiatric Disorders: A Literature Review and Look into Future Prospects," Musical Offerings: Vol. 10 : No. 2 , Article 2.

DOI: $10.15385 / j m o .2019 .10 .2 .2$

Available at: https://digitalcommons.cedarville.edu/musicalofferings/vol10/iss2/2 


\title{
The Effects of Music Therapy on Individuals Suffering from Psychiatric Disorders: A Literature Review and Look into Future Prospects
}

\section{Document Type}

Article

\begin{abstract}
Music therapy is a field of psychology and psychotherapy that exists under the broad field of study known as expressive arts therapy. This form of therapy is experiential in nature, and it gives participants vehicles through which they can tell their stories and share their emotions while actively exploring their thoughts. Music therapy has proven to be an effective treatment for a myriad of psychological disorders, including anxiety, depression, bipolar disorder, PTSD, and schizophrenia. In order to grant a base level of understanding, research was conducted examining the methods and approaches used in music therapy, along with the psychological and physiological effects that it has on its participants. Primary findings show that the trend in research is toward a more broad-spectrum use of music therapy, as well as an expansion of the current treatment contexts and conventions. Secondary findings revolve around the limited literature regarding music therapy and its association with those who suffer from psychiatric disorders and are already receiving pharmacologic treatment. Tertiary findings will revolve around the discovery of various neurobiological and neurophysiological findings related to music therapy and its effects on the brain.
\end{abstract}

\section{Keywords}

Music therapy, psychology, mental health, depression, anxiety, PTSD, psychiatry, music

\section{Creative Commons License}

\section{(1) (9)}

This work is licensed under a Creative Commons Attribution-Noncommercial-No Derivative Works 4.0 License. 


\title{
The Effects of Music Therapy on Individuals Suffering from Psychiatric Disorders: A Literature Review and Look into Future Prospects
}

\author{
Zachary J. Krauss \\ Cedarville University
}

$\mathrm{M}$ usic therapy is a relatively recent development in the world of psychology, and it is certainly a recent development in the world of medicine. Regardless, some of the earliest references to music therapy appeared in a 1789 article titled "Music Physically Considered," which discussed therapeutic value of music that was referenced in two different medical dissertations (AMTA, 2019).*

The value of music therapy as a legitimate form of psychotherapy has been debated in the past few years. A quick search through the current literature available on music therapy on PubMed yields resources that both support and deny the efficacy of music therapy. However, upon closer inspection, it seems that the more recent research in the field of music therapy is finding that this form of therapy truly is a viable treatment method for those in need. This paper argues that, while the effectiveness of music therapy has been debated in the past, recent literature shows that music therapy is significantly more effective in practice than psychotherapy or psychological pharmacotherapy alone.

This paper serves as a review of recent literature (published between 2014 and 2019) on the effectiveness of music therapy in clinical settings, with a focus on the effectiveness of music therapy to treat those with psychiatric disorders. A narrower focus was utilized to accommodate articles and reviews that included information about music therapy and

Musical Offerings 10, no. 2 (2019): 61-80

ISSN 2330-8206 (print); ISSN 2167-3799 (online)

(C) 2019, Zachary J. Krauss, licensed under CC BY-NC-ND

(http://creativecommons.org/licenses/by-nc-nd/4.0/)

* Due to the scientific content of this paper, the author has chosen to use APA style, and the Editors have concurred. 
its effect on patients already taking psychiatric medications in order to treat the symptoms of their disorder. The paper is organized by disease states such as depression, bipolar disorder, and post-traumatic stress disorder (PTSD).

\section{Depression and Anxiety}

The American Psychiatric Association defines depression (major depressive disorder) as a "common and serious medical illness that negatively affects how you feel, the way you think and how you act" (Parekh, 2017c). Depression is mainly characterized by feeling sad or having a depressed mood, losing interest in previously enjoyable activities, loss of energy or increased fatigue, and even thoughts of death or suicide. The American Psychiatric Association also defines anxiety disorders as those disorders that differ from normal feelings of nervousness or anxiousness and involve excessive fear and anxiety (Parekh, 2017a). Types of anxiety disorders include generalized anxiety disorder, social anxiety disorder, and panic disorder. Treatment for depression and anxiety usually includes cognitive behavioral therapy as well as other forms of psychotherapy, and sometimes antidepressants or antianxiety medications, respectively.

In a 2018 study on anxiety and depression levels in Alzheimer's patients receiving several music therapy sessions lasting about one hour each, patients were shown to experience decreasing levels of stress as well as significantly lowered levels of depression and anxiety. During their sessions, patients were invited to sing an introductory song before being led in a song related to flowers. For these patients, the song on flowers functioned to improve base cognitive levels, memory, and lyric recall, while also strengthening the temporal link between flowers and positive emotions associated with the season of spring. (Ortí et al., 2018).

Enzyme-Linked ImmunoSorbent Assay (ELISA) immunoassay technique was used in order to measure the salivary levels of cortisol, and general psychological state was measured using the Hospital Anxiety and Depression Scale. The study showed that after music therapy, patients' cortisol levels decreased, which is relevant considering recent research which has shown that cortisol is significantly associated with increasing levels of depression (Keller et al., 2017). Not only did the research of Ortí et al. (2018) solidify the previous research on cortisol and its effect on patients' depression, but it specifically showed that 
those suffering from depression within the context of another comorbid (co-occurring) disorder can still be treated with music therapy.

In another study, Fernandes and D'silva (2019) researched the effects of music therapy on hemodialysis patients. One of the most common psychological disorders affecting those undergoing hemodialysis is depression. This study utilized passive music therapy in which the patients were exposed to music of an unspecified genre in order to affect their global state (one accounting for all mental and emotional factors). Using qualitative data from the Depression Anxiety Stress Scale (DASS) tool, 40 subjects were analyzed. The therapy was shown to be effective in decreasing general stress, anxiety, and depression levels, all significant up to a $95 \%$ confidence interval. This research proves that music therapy can be effective in preventing symptoms of depression from becoming exacerbated during times of treatment, giving music therapy a viable entrance into the medical realm rather than simply the context of psychotherapy (Fernandes \& D'silva, 2019).

One article investigating the effects of music on those with generalized anxiety disorder found that music therapy was effective at reducing anxiety as well as depression levels in those with the disorder. The patients in the study experienced both receptive and active music therapy. During the receptive portion, they listened to music without active involvement, while the active portion involved interacting with a music therapist to compose music/songs. This study utilized listening to an unspecified genre of music without therapist intervention, as well as active music therapy treatment in which the therapist guided the patient in producing music using their voices, bodies, and/or musical instruments. The active and passive music therapies were not directly related in the study, but the patients likely benefited from the combined nature of both kinds of therapy (Gutiérrez \& Camarena, 2015).

The researchers acknowledge that this was a small pilot study, but they do suggest that music therapy could effectively function as a psychotherapeutic treatment for psychiatric care of those with generalized anxiety disorder, even to the point of being considered before some pharmacologic treatments (Gutiérrez \& Camarena, 2015).

Another study looking at music therapy in a medical context looked at the effects of music therapy on anxiety and depression in cancer patients. This is very significant considering that up to $75 \%$ of cancer patients in late stages of their illness experience either depression or anxiety 
(Jasemi, Aazami, \& Zabihi, 2016). The study by Jasemi et al. (2016) found that in the group experiencing music therapy intervention, levels of anxiety and depression were decreased.

The study utilized a very passive form of music therapy in which patients listened to light and peaceful music for at least 20 minutes per day intentionally for 3 days. This therapy was implemented in a group setting, so adherence was guaranteed in all scenarios. The study found that there was a significant decrease in levels of depression and anxiety in the group that experienced the intervention. While the sample size for this study is small and future research on the effects of music therapy on cancer patients is needed, this research is helpful for showing the clinical relevance of music therapy for patients who are terminally ill (Jasemi et al., 2016).

In a systematic review evaluating the effects of music therapy on preand post-operative states of pediatric patients, there was a substantial amount of research showing that music therapy is effective for relieving fear, decreasing anxiety, and acting as an alternative for pharmacological treatment (van der Heijden, Araghi, van Dijk, Jeekel, \& Hunink, 2015).

Two studies discussed in the review found that music as an intervention method had significant pain-reducing effects on participants, and another study found non-statistically significant levels of pain-reducing effects. Much of the data collected by van der Heijden et al. (2015), even that which had to be excluded, found that post-operative music interventions helped to reduce subjective levels of pain. Pain was measured using the Visual Analogue Scale, the Coloured Analogue Scale, and the Facial Pain Scale. Music therapy interventions examined in the study involved either live music performance by a music therapist or musical creation therapy in which the patient assisted with creation of melodies.

A study looking specifically at the neurobiology of music therapy found that the anti-anxiety effect caused by the intervention of music is associated with glutamic acid and GABA (Gamma-aminobutyric acid) levels in the hippocampus (Chen, 2018). Although this study focused on a murine (mouse) model and no in-person human trials were done, the results were still significant. The subjects were exposed to classical and rock music (a secondary hypothesis was that classical music by Mozart would be more effective) for about one hour each day for 21 days. 
It was discovered in the same study that music therapy can improve anxiety behavior, creating an easing effect on the emotional state of those affected. Music appeared to have a regulatory effect on the levels of glutamic acid and GABA in the hippocampus, functioning to balance the excitatory and inhibitory function and working as a mood stabilizer (Chen, 2018). The mice seemed to be more capable to complete a maze that otherwise might have proven difficult in an anxious state; levels of GABA and glutamic acid were directly measured from the hippocampus of the mice after their death. On a more qualitative note, music by Mozart seemed to be more effective than rock music at decreasing anxiety. This research shows great promise for future research into music therapy's effect on the physiology of the brain, and for potential specifically aimed forms of intervention.

\section{Trauma-Related Disorders}

Trauma related disorders include acute traumatic stress disorder, posttraumatic stress disorder (PTSD), and dissociative disorders related to trauma. These disorders' symptoms are fairly serious, and effects on the individual tend to be debilitating. PTSD is defined by the American Psychiatric Association as a "psychiatric disorder that can occur in people who have experienced or witnessed a traumatic event such as a natural disaster, a serious accident, a terrorist act, war/combat, rape or other violent personal assault" and was the disorder referenced most frequently in the literature (Parekh, 2017d). The American Psychiatric Association continues to describe the symptoms of PTSD as consisting of intense, disturbing thoughts and feelings related to a traumatic event that last long after that event has ended. They state that PTSD patients often continue to experience the event through nightmares, flashbacks, or intrusive memories, and experience symptoms such as sadness, fear/anxiety, or anger, as well as a general detachment from reality (Parekh, 2017d).

Treatment for PTSD often involves cognitive processing therapy, group therapy, prolonged exposure therapy (exposing patients to flashbackinducing stimuli in a safe environment in order to encourage healing), and medications such as SSRIs (selective serotonin reuptake inhibitors) and SNRIs (selective norepinephrine reuptake inhibitors). Medications for anxiety or physical agitation are also frequently used.

One study conducted by Metzner, Verhey, Braak, and Hots (2018) focused on the effects of music therapy on PTSD patients who had 
suffered from torture, political violence, and flight. The study showed that patients had a "pathological perception of loudness." Most listeners in the study suffered from hearing loss of some sort but still reacted positively to music therapy most of the time.

This study focused primarily on the kinds of sounds that negatively affected those in trauma therapy, with the intent of discovering what kinds of sounds, beats, and rhythms to avoid in music therapy. The study also revealed the general effectiveness of the therapy. Interventions involved in the study included passive listening to music, as well as certain exercises involving the patients themselves learning percussion; patients' individual utterances of need were used in order to determine specific implementations of music therapy. The researchers gathered qualitative data by asking the patients questions about how their mental state was affected, and psychoacoustic measurements ascertaining levels of most comfortable loudness (MCL) were used to examine the effect of harsh sounds on trauma. Metzner et al. (2018) found that once a perception to loudness and discordant sounds was acknowledged and accounted for, music therapy was effective for a majority of patients.

Metzner et al. (2018) also posited that patients with complex PTSD found ways through their own personal coping to deal with unpleasant sounds that evoke flashbacks or traumatic memories. The results of the findings suggested that, rather than eliminating music therapy from practice because of its potential to trigger patients, music therapy, especially for those with PTSD, must be fine-tuned to the individual in order to discover what kinds of sounds and beats are acceptable and truly beneficial.

A research design pilot study by Pezzin, Larson, Lorber, McGinley, and Dillingham (2018) performed research evaluating the effect of music therapy on PTSD patients. The music therapy intervention in this study focused on music instruction in order to help veterans learn how to better cope with their trauma. Veterans were given guitar instruction in hourlong individual lessons that lasted for at least six sessions. Along with these individual interventions, veterans were required to attend at least three group sessions in which they would discuss not only their music therapy, but anything else related to their disorder and trauma.

A follow-up interview was utilized in order to determine PTSD symptomology. Patients took a self-test known as the 'PTSD Checklist Civilian' (PCLC) to measure PTSD presence and severity. The study 
showed that active music instruction in the form of music therapy combined with group therapy discussions holds promise as a complementary PTSD treatment method in combination with either psychotherapy or pharmacotherapy. The results showed that this form of treatment was extremely effective as a method of emotional expression to decrease psychiatric symptoms in a myriad of contexts (Pezzin et al., 2018). The researchers believe that a program such as this could be a viable option for engaging such a vulnerable population.

One study on the effects of creative art therapies in general (including dance, music, art, and drama therapies) found that PTSD patients had potential to be greatly benefited by music therapy (Baker, Metcalf, Varker, \& O'Donnell, 2018). One of the randomized control trials listed in the review found that a music therapy regimen involving improvisation led to a significant reduction in PTSD symptoms compared to controls. Patients were encouraged to work with the music therapist in order to produce vocal music accompanied with an instrumental track of their choice.

Not only did this active form of music therapy prove efficacious, but another study listed in the review showed that passive listening to relaxing music didn't seem to have any significant effects on PTSD symptoms, implying that active music therapy is necessary in order to evoke change in PTSD patients (Baker et al., 2018). The review concluded that music and art therapy showed promise for effectively decreasing major symptoms of PTSD. The review examined randomized control trials or controlled studies in which PTSD symptom severity or diagnosis were in some way quantitatively measured. The researchers posited that the "specialist skills and ongoing therapeutic relationship is a key factor to reducing symptoms of PTSD," reaffirming the significance of the unique music therapist/patient relationship in traumarelated treatment through musical intervention. (Baker et al., 2018, p. 649).

An article on ICU-related PTSD explored the concept of songwritingbased music therapy as an option to reduce the negative effects of the trauma sometimes encountered in an intensive care unit. ICU-related PTSD is caused by the trauma and discomfort associated with medical practices such as ventilation adjustments, lapses in anesthesia and/or analgesia, prolonged sedation, restraint usage, prolonged time spent in the hospital itself, and opioid-induced hallucinations (Noyes \& Schlesinger, 2017). 
In the study, Noyes and Schlesinger (2017) state that they are hopeful music therapy could change the prognosis of ICU-related PTSD, positively influencing those affected by ICU-related trauma. In the article, positive effects of songwriting include mediating the isolated world of the ICU, alleviating stress and anxiety, and enabling communication, relaxation, and expression in patients who might otherwise have no outlet. The study analyzed responses to qualitative follow-up interviews as well as global state perceptions from attending health professionals. Patients were exposed to passive/receptive music while in the ICU and were encouraged to write lyrics and basic melodies once in recovery or in less critical states in the hospital.

Another review study examining the effect of music therapy on the emotions of trauma showed that music has the unique quality of touching "multiple symptomatic clusters that [are] present in patients with trauma and PTSD" (Landis-Shack, Heinz, \& Bonn-Miller, 2017, p. 338). The study found that, while music therapy was effective at treating all the symptom clusters examined in the study (including arousal/reactivity, avoidance, intrusions, and negative cognition/mood), it was most effective at remedying negative cognition/mood.

The review posited that music therapy as a tool can directly address and prevent/reduce certain debilitating symptoms. The primary outlook the researchers had for music therapy in the review was the quality of music therapy in its ability to encourage resilience. The intervention they utilized involved patients producing music and explaining why they wrote the lyrics that they did, or why they chose the melodies that they did. Landis-Shack et al. (2017, p. 338-339) stated that music therapy has a great amount of promise to "help trauma-exposed individuals harness their ability to recover elements of normality in their life following great adversity."

\section{Schizophrenia and Personality Disorders}

The American Psychiatric Association defines schizophrenia as a chronic brain disorder that, when active, is characterized by symptoms including "delusions, hallucinations, trouble with thinking and concentration, and lack of motivation" (Parekh, 2017e). Personality disorders are defined as a "way of thinking, feeling and behaving that deviates from the expectations of the culture, causes distress or problems functioning, and lasts over time" (Robitz, 2018). 
While schizophrenia and personality disorders are substantially different in their symptoms and manifestations, much of the literature seems to gather the two disorders together for testing and research purposes. Consequently, they will be examined together for the purpose of this review. The treatments for schizophrenia and personality disorders include antipsychotic drugs and cognitive behavioral therapy, as well as dialectical behavior therapy.

One study looking at music therapy and schizophrenic/personality disorder patients found that music therapy seemed to create a positive bond between the patient and the counselor (Hannibal, Pedersen, Hestbæk, Sørensen, \& Munk-Jørgensen, 2014). This data is hopeful because, for patients with personality disorders and schizophrenia, psychotherapy and counseling are often rendered less effective as the connection between counselor and patient is often unable to become strong enough in order to enact true change. Trust is a critical factor in counseling, especially for patients who often dissociate from the rest of society, and this study found that patients were more liable to trust after active music therapy sessions were implemented.

The intervention allowed patients to speak with their therapist about why they enjoy certain forms of music, and, after more in-depth discussion, the music therapist worked with the patients to help them improvise a piece or set of small pieces in order to provide an emotional outlet. Psychiatric and psychological data were collected during an observational period by the researchers for one year, along with a yearlong follow-up observation, and the study found that $82 \%$ of patients remained in treatment. The researchers attributed this positive result to an alliance that "may be possible despite general severity of illness" (Hannibal et al., 2014, p. 378). This further emphasizes the importance of trust in the relationship and implies that music therapy creates a unique bond that is harder to establish through other psychotherapy techniques (Hannibal et al., 2014).

An article researching the effects of music therapy on borderline personality disorder found similar results to those of Hannibal et al. regarding the relationship of the therapist with the patient. This study by Strehlow and Lindner (2016, p. 154) discussed the fact that music therapy seemed to help "as a third element to regulate the relationship between patient and therapist" where borderline personality patients often have difficulty in relationships as a result of regulating their own proximity and distance, etc. 
Findings in this qualitative study were measured by case study comparisons conducted by the researchers. These case studies showed that, especially with borderline personality disorder, the process of applying music therapy is complex and involved, particularly due to the difficulty of borderline personality disorder patients to deal with extreme emotions and feelings. Music therapy has the ability to bring out emotions that a patient might not have previously processed in psychotherapy as well as the potential to heighten other emotions that might have otherwise been muted. However, the study also found that, when approached correctly, patients can benefit from the therapy in a way they might not have benefitted from any other form of counseling. The correct approach implied in this research seemed to predominantly revolve around client comfort and the individual nature of care (Strehlow \& Lindner, 2016).

A systematic review compiled by the Cochrane Library on the effect of music therapy on schizophrenia and similar disorders discussed the broad data in the literature very concisely (Mossler, Chen, Heldal, \& Gold, 2014). The review surveyed randomized controlled trials and controlled studies, searching for those which had positive and significant data. One finding of the review was that music therapy apparently had a very strong effect on the global state of those involved. Patients involved in these results were given music therapy in high-dose (more than 15 sessions over the scope of the trial) format, so it is unclear if any lesser amount of music therapy would be as effective.

Another finding of the review involved assessing the general mental state of those involved. In doing so, it discovered that low-dose music therapy (of less than 20 minutes in a session) didn't seem to have significant results; however, it was apparent that medium- and high-dose variants were effective. The same discrepancy between high and low dosing was observed in measurements regarding cognitive and emotional functioning (Mossler et al., 2014).

The conclusion of the study stated that music therapy, in addition to standard care, can help people with schizophrenia improve their global state, negative symptoms, depression, anxiety, and social functioning over the short- to medium-term. Music therapy seems to address especially motivational, emotional, and relational aspects, and help patients reconnect to both intrapersonal and social resources. The researchers involved in the review acknowledged that, in order for the positive effects to be realized in the patients, at least 20 sessions are 
needed, and active participation (as opposed to passive music therapy) is essential (Mossler et al., 2014).

Important to note for the previously mentioned review is that a majority of the examined studies included patients who were already taking either a mood stabilizer or an antipsychotic, emphasizing the ability of music therapy to help even those already on prescribed psychological medications. Geretsegger et al. (2017) posited that this kind of result, especially for those suffering from extremely hard-to-manage schizophrenia, could be beneficial for future research into new methods of treatment. While it doesn't appear that being on medications for the disorder is required for the music therapy to work, it seems that music therapy is not hindered by the presence of most antidepressants or antianxiety medications, nor by most mood stabilizers or antipsychotics.

\section{Bipolar Disorder}

Bipolar disorder is defined by the American Psychiatric Association as a set of "brain disorders that cause changes in a person's mood, energy and ability to function...People with bipolar disorders have extreme and intense emotional states that occur at distinct times, called mood episodes" (Parekh, 2017b).

Bipolar is separated into three categories: Bipolar I, Bipolar II, and Cyclothymic Disorder. Each of these distinctions imply different levels and cycles of mood changes and swings, but the concept of emotional and mood inconsistencies remains the same throughout. Treatment for bipolar disorders often involves either psychotherapy or medications such as mood stabilizers and antidepressants. Which medications or types of psychotherapy are utilized varies based on individual symptomatic presentation.

One study looking at the effects of music therapy on bipolar disorder patients in a euthymic (normal mood) state found that music created more complex negative emotions in those responding to subjectively uplifting music (Choppin et al., 2016). Data was collected from participants using two self-reported scales (ERS and MAThyS), along with scales that required them to rate their music-induced emotions. While this initially seems to imply that music therapy has a negative effect on patients, the data found that the patients' response to the therapy did not differ in their subjective feelings compared to the control group. 
All patients involved in the study were taking mood stabilizers, and almost $25 \%$ of them were taking antidepressants.

One hypothesis for these results was that patients with bipolar disorder might respond in this way as a result of their struggle to regulate their own positive emotions, which creates a form of distress manifested as a negative emotion. While the data says little for the effectiveness of music listening alone on bipolar disorder, the research group posited that listening to music could be a beneficial tool in allowing patients to better understand their emotions and the roots therein (Choppin et al., 2016).

A review article considering the effect of music therapy on psychiatric patients and their already-established medical regimen found that music therapy could be extremely beneficial in the treatment of patients exhibiting psychotic symptoms, including bipolar patients. Results were measured by medical professionals as they observed the continuous effect of the participants' medications (Stefani \& Biasutti, 2016). The article found that even patients with bipolar disorder who were taking multiple kinds of psychological drugs could still be positively affected by music (the positive influence on psychotic symptoms seen in patients was not hindered by pharmacological treatment).

\section{Broad Contexts}

One systematic review done on patients undergoing treatment for substance abuse disorders looked at the effect of music therapy on the patients' psychiatric symptoms and outcomes. The review examined the effects of music therapy on many different variables, concentrating on randomized controlled trials. Interestingly, many of the patients involved in this extensive overview of over 40 articles were taking psychiatric medications such as mood stabilizers, antidepressants, benzodiazepines, and antipsychotics (Hohmann, Bradt, Stegemann, \& Koelsch, 2017).

A majority of the articles viewed in the Hohmann et al. (2017) review found that music therapy was helpful for them as a supplement to their treatment, and it also showed in some studies that music therapy was effective in supplying patients with more sufficient motivation and hope. While symptoms like depression, withdrawal/craving, coping skills, and participation could not be significantly correlated with music therapy, the review was able to correlate music therapy and music interventions positively with anxiety, medical symptoms, sadness, anger, and stress. These data are helpful in the understanding of music therapy and its 
effectiveness not only in patients already undergoing other forms of psychotherapy/pharmacotherapy, but also in examining patients suffering from a unique comorbid condition in the form of substance abuse disorder.

In a study examining the effects of music therapy on children and adolescents suffering from behavioral and emotional problems, findings demonstrated that both active and receptive music therapy had the ability to improve outcomes such as social skills and communication in patients with difficulties in those areas due to emotional and developmental impediments (Porter et al., 2017). Data was collected using a Social Skills Improvement System Rating Scales (SSIS) survey as a follow-up after 13 weeks of the study. The data showed potential for the effectiveness of music therapy in treating self-esteem issues and depression in youth with certain deficits.

The data by Porter et al. (2017) also implied that music therapy would be effective in clinical settings for children and adolescents suffering from comorbid mood and development disorders. The research team concluded that a substantial amount of the positive effect of music therapy observed in the study was a result of the care and attention given by the therapist in the music therapy relationship, again pointing to the significance of a unique relationship created between a music therapist and patient.

A study conducted on a group of patients with neurologic disorders (primarily Alzheimer's and stroke) found that music therapy could be helpful in not only reducing "psychological and behavioral disturbances related to neurological disorders," but also for "promoting the functional recovery" (Raglio et al., 2015, p. 75). The study reviewed articles that had quantitative and qualitative responses from health professionals related to the symptoms and severity of the examined disorders. The article focused on mood symptoms, specifically depression and hopelessness, which are often experienced by patients with neurological illness.

It was found by Raglio et al. (2015) that, especially for stroke patients with depressive symptoms, music therapy was beneficial for improving mood and encouraging general quality of life. It was also shown that music therapy improved all levels of anxiety to some extent. This once again shows that, for those in comorbid situations where mental 
symptoms are paired with other disorders or illnesses, music therapy can be effective in combination with other forms of intervention.

\section{Brain Chemistry, Music, and Neuroscience}

The mechanisms of music therapy are discussed differently in many of the articles that were reviewed for this paper. While several theories and ideas have been proposed to explain music therapy, it doesn't appear that any one particular thought system is currently dominating the field. Recently, it seems that a focus on neurobiology and neuroplasticity is present in the literature, and that focus will be discussed here.

In an article by Constantin (2018, p. 19), music therapy was explained in the concept of neuroplasticity. As defined in the article, neuroplasticity is "the ability of the brain to change throughout a person's life span as a consequence of sensory input, motor action, reward, or awareness." The article goes on to explain the many ways that music therapy fits into this concept.

Reward circuitry in the brain focuses on research made in psychology and neurophysiology proving that dopamine, an important neurotransmitter, is associated with neuroplasticity and changes in the brain. An article on reward circuitry and addiction discusses the fact that normal neuronal activity naturally associates a "reward" circuitry to everyday tasks such as eating, sex, or social interaction. Things like addiction, trauma, and the reversal of these states are the focus of more recent brain circuitry research. (Cooper, Robison, \& Mazei-Robison, 2017).

One way that Constantin (2018) explains neuroplasticity and music therapy is as a reward system. By comparing music therapy to food or drugs in the realm of circuitry, Constantin (2018) is able to argue that music has the potential to drastically affect neuroplasticity.

Another concept Constantin (2018) brings into music's effect on the brain is a theory known as 'The Hebbian Principle,' which basically states that in order for strengthening of a current connection in the brain to occur, or in order for a new connection to be made, two neurons have to start their action potentials (or their electrical "firing") at the same time. Constantin (2018) presents research showing that rhythm/beat in music is often crucial to music therapy since it is effective in linking human behaviors to neural networks. Music therapy also linked human 
behaviors to activities such as dancing and singing, while even affecting autonomic nervous system processes like breathing, heart rate, and sleep cycles (Constantin, 2018).

Constantin $(2018$, p. 23) states that musical training clearly leads to changes in the human brain. In a section on how neuroplasticity applies directly to music therapy, the article argues that pairing music preferred by a patient with the emotions they express while composing and experiencing music creates a kind of "synchronized dopaminergic reward that may strengthen the connections associated with related emotional expression while suppressing connectivity associated with the unrelated one."

Constantin (2018, p. 24) concludes her review of research on neuroplasticity by stating that "music is a tool that enhances neuroplasticity in the brain. Through music therapy non-musical behavior could change, the processes governing the change in behavior leading to changes in the brain. The potential of music therapy to change neural connectivity... underlie the positive effects of music therapy."

In an article about the neurobiological foundations for music therapy, the concepts of rhythm and neural entrainment are considered very important factors that play into music therapy's effect on the brain (Thaut, McIntosh, \& Hoemberg, 2015). This article focuses on research that began in the 1990s and has continued until very recently, proving that music and the rhythm it holds are directly related to sensory and motor pathways in the brain. The researchers posit that, as a result of the "entrainment" of music therapy patients with the music they are listening to, music therapy can assist in rehabilitation both emotionally and physiologically.

The article states that music therapy as a field has great capability to begin shifting into a more neurologic context, meaning that it could treat not only emotional and psychiatric disorders, but also neurologic disorders and motor disorders. Music therapy, according to this source, provides the ability, in a way, to "rewire" patients with movement or speech disorders in a way that would allow them to once again find their "rhythm" (Thaut et al., 2015).

The authors hope that this research will allow music therapists to gain a better understanding of how music therapy can fit into the concepts of executive and psychosocial function, attention, and memory when it 
comes to psychiatric treatment. They claim that while these areas haven't had much success in the past as far as music therapy interventions were involved, this new knowledge could help researchers better understand music and rhythm in a therapeutic context in order to focus on certain psychiatric deficits (Thaut et al., 2015).

Another current focus in the realm of music therapy is the actual neurobiological and chemical changes that are imparted by music therapy. A study by Keeler et al. (2015) looked specifically at the effects of singing on two chemicals, ACTH and oxytocin. ACTH, or adrenocorticotropic hormone, is a neurohormone that is associated with the release of glucocorticoids like cortisol from the endocrine system. Oxytocin is a neuropeptide that is released from the pituitary gland as a hormone, where it colocalizes with stress hormones and helps to suppress the HPA axis and mediate human social behavior.

While the study's sample size was relatively small, the researchers were able to collect significant statistical data from blood levels of ACTH and oxytocin, showing that music therapy was effective at decreasing and increasing the levels of these neurochemicals, respectively. This implies that there are direct effects of music therapy on the immediate neurochemistry of the brain and the rest of the body as a result of the release of hormones from the brain which can affect the endocrine system, the immune system, and the cardiovascular system (Keeler et al., 2015). Interesting but not conclusive in this study is the fact that oxytocin is significant in the chemistry of human bonding. Further research on music therapy's role in increasing oxytocin levels could help yield a better understanding of the benefits of the relationship between the patient and the music therapist, building on the precedent clearly set in the literature.

\section{Conclusion}

The literature present on music therapy from roughly the past five years clearly shows that music therapy can be effective as a treatment method for various forms of psychiatric disorders. Whether combined with pharmacotherapy or not, music therapy's positive effects are evident. It seemed apparent in the literature that active music therapy as opposed to passive or receptive variants was the most effective, especially for the treatment of more serious symptoms; however, many of the articles acknowledged that more research was needed in this area before definitive statements could be made. 
This review also discussed some of the recent theories and ideas circulating in the actual action of music therapy in the brain. Much of the research in the field is moving toward a focus on concepts like neuroplasticity and brain circuitry, indicating that music can have a significant and lasting effect on the brain, rather than just on mood or emotional responses. At the same time, it seems that, especially for music therapy to be applied in medical contexts, the upcoming concept of rhythmic entrainment gives positive implications for music therapy's use not only for neurologic and motor disorders, but also for more specific and debilitating aspects of serious psychiatric disorders such as dissociation and memory loss. Brain chemistry associated with certain neurohormones and neuropeptides has also been examined in recent studies which seem to show promise in gaining a better understanding of the general molecular microenvironment created by the context of music therapy.

The results of this literature review all describe a more positive understanding of music therapy and its effects on those suffering from mood symptoms and psychiatric disorders. The literature points to music therapy being implemented in many different varieties of practice settings, implying a need for future research in those areas, and the current research on the actual effects of music therapy on the brain also begs for future research to be carried out.

\section{References}

Baker, F.A., Metcalf, O., Varker, T., \& O’Donnell, M. (2018). A systematic review of the efficacy of creative arts therapies in the treatment of adults with PTSD. Psychological Trauma: Theory, Research, Practice, and Policy 10(6), 643-651. doi: $10.1037 /$ tra0000353.

Chen, R. (2018). Emotional inhibitory effect of music therapy on anxiety neurosis based on neural content analysis in hippocampus. NeuroQuantology 16(5), 53-59. doi:10.14704/nq.2018.16.5.1431.

Choppin, S., Trost, W., Dondaine, T, Millet, B., Drapier, D., Verin, M., ...Grandjean, D. (2016). Alteration of complex negative emotions induced by music in euthymic patients with bipolar disorder. Journal of Affective Disorders 191, 15-23. doi:10.1016/j.jad.2015.10.063. 
Constantin, F.A. (2018). Music therapy explained by the principles of neuroplasticity. Bulletin of the Transilvania University of Braşov 11(60). doi:10.14510/araj.2017.4113.

Cooper, S., Robison, A.J., \& Mazei-Robison, M.S. (2017). Reward circuitry in addiction. Neurotherapeutics 14(3), 687-697. doi:10.1007/s13311-017-0525-z.

Fernandes, S.T. \& D'silva, F. (2019). Effectiveness of music therapy on depression, anxiety and stress among haemodialysis patients. International Journal of Nursing Education 11(1), 124-129. doi:10.5958/0974-9357.2019.00024.2.

Geretsegger, M., Mössler, K.A., Bieleninik, Ł., Chen, X.J., Heldal, T.O., \& Gold, C. (2017). Music therapy for people with schizophrenia and schizophrenia-like disorders. The Cochrane Database of Systematic Reviews 5(5), CD004025. doi:10.1002/14651858.CD004025.pub4.

Gutiérrez, E.O.F., \& Camarena, V.A.T. (2015) Music therapy in generalized anxiety disorder. The Arts in Psychotherapy 44, 19-24. doi:10.1016/j.aip.2015.02.003.

Hannibal, N., Pedersen, I.N., Hestbæk, T., Sørensen, T.E., \& MunkJørgensen, P. (2012). Schizophrenia and personality disorder patients' adherence to music therapy. Nordic Journal of Psychiatry 66, 376-379. doi:10.3109/08039488.2012.655775.

Hohmann, L., Bradt, J., Stegemann, T., \& Koelsch, S. (2017). Effects of music therapy and music-based interventions in the treatment of substance use disorders: A systematic review. PLoS One 12(11). doi:10.1371/journal.pone.0187363.

Jasemi, M., Aazami, S., \& Zabihi, R.E. (2016). The effects of music therapy on anxiety and depression of cancer patients. Indian Journal of Palliative Care 22, 455-458. doi:10.4103/0973-1075.191823.

Keeler, J.R., Roth, E.A., Neuser, B.L., Spitsbergen, J.M., Waters, D.J.M., \& Vianney, J. (2015). The neurochemistry and social flow of singing: Bonding and oxytocin. Frontiers in Human Neuroscience 9(518). doi:10.3389/fnhum.2015.00518.

Keller, J., Gomez, R., Williams, G., Lembke, A., Lazzeroni, L., Murphy, G.M., Jr., \& Schatzberg, A.F. (2017). HPA axis in major depression: Cortisol, clinical symptomatology and genetic variation predict cognition. Molecular Psychiatry 22, 527-536. doi: $10.1038 / \mathrm{mp} .2016 .120$. 
Landis-Shack, N., Heinz, A.J., \& Bonn-Miller, M.O. (2017). Music therapy for posttraumatic stress in adults: A theoretical review. Psychomusicology: Music, Mind, and Brain 27(4), 334-342. doi:10.1037/pmu0000192.

Metzner, S., Verhey, J., Braak, P., \& Hots, J. (2018). Auditory sensitivity in survivors of torture, political violence and flight: An exploratory study on risks and opportunities of music therapy. The Arts in Psychotherapy 58, 33-41. doi:10.1016/j.aip.2018.02.001.

Noyes, E.M., \& Schlesinger J.J. (2017). ICU-related PTSD: A review of PTSD and the potential effects of collaborative songwriting therapy. Journal of Critical Care 42, 78-84. doi:10.1016/i.jerc.2017.06.014.

Ortí, J.E.R., García-Pardo, M.P., Iranzo, C.C., Madrigal, J.J.C., Castillo, S.S., Rochina, M.J., \& Gascó, V.J.P. (2018). Does music therapy improve anxiety and depression in Alzheimer's patients? The Journal of Alternative and Complementary Medicine 24(1), 33-36. doi:10.1089/acm.2016.0346.

Parekh, R. (2017). What are anxiety disorders? Retrieved from https://www.psychiatry.org/patients-families/anxietydisorders/what-are-anxiety-disorders.

Parekh, R. (2017). What are bipolar disorders? Retrieved from https://www.psychiatry.org/patients-families/bipolardisorders/what-are-bipolar-disorders.

Parekh, R. (2017). What is depression? Retrieved from https://www.psychiatry.org/patients-families/depression/ what-is-depression.

Parekh, R. (2017). What is posttraumatic stress disorder? Retrieved from https://www.psychiatry.org/patients-families/ ptsd/what-is-ptsd.

Parekh, R. (2017). What is schizophrenia? Retrieved from https://www.psychiatry.org/patients-families/schizophrenia/ what-is-schizophrenia.

Pezzin, L.E., Larson, E.R., Lorber, W., McGinley, E.L., \& Dillingham, T.R. (2018). Music-instruction intervention for treatment of post-traumatic stress disorder: A randomized pilot study. BioMed Central Psychology 6(60). doi:10.1186/s40359-018-0274-8. 
Porter, S. McConnell, T., McLaughlin, K., Lynn, F., Cardwell, C., Braiden, H., ...Holmes, V. (2017). Music therapy for children and adolescents with behavioural and emotional problems: A randomised controlled trial. The Journal for Child Psychology and Psychiatry 58(5), 586-594. doi:10.1111/jcpp.12656.

Raglio, A., Attardo, L., Gontero, G., Rollino, S., Groppo, E., \& Granieri, E. (2015). Effects of music and music therapy on mood in neurological patients. World Journal of Psychiatry 5(1), 68-78. doi:10.5498/wjp.v5.i1.68.

Robitz, R. (2018). What are personality disorders? Retrieved from https://www.psychiatry.org/patients-families/personalitydisorders/what-are-personality-disorders.

Stefani, M.D., \& Biasutti, M. (2016). Effects of music therapy on drug therapy of adult psychiatric outpatients: A pilot randomized controlled study. Frontiers in Psychology 7(1518). doi:10.3389/fpsyg.2016.01518.

Strehlow, G. \& Lindner, R. (2016). Music therapy interaction patterns in relation to borderline personality disorder (BPD) patients. Nordic Journal of Music Therapy 25(2), 134-158. doi:10.1080/08098131.2015.1011207.

Thaut, M.H., McIntosh, G.C., \& Hoemberg, V. (2015).

Neurobiological foundations of neurologic music therapy: Rhythmic entrainment and the motor system. Frontiers in Psychology 5(1185), 1-6. doi:10.3389/fpsyg.2014.01185.

van der Heijden, M., Araghi, S.O., van Dijk, M., Jeekel, J., \& Hunink, M.G.M. (2015). The effects of perioperative music interventions in pediatric surgery: A systematic review and meta-analysis of randomized controlled trials. PLoS One 10(8). doi:10.1371/journal.pone.0133608. 